Case Report

\title{
Spinal Cord Glioneuronal Tumor with Rosetted Neuropil-Like Islands in Pediatric Age Group
}

\author{
Nil Comunoglu, ${ }^{1}$ Ozgur Kilickesmez, ${ }^{2}$ and Buge $\mathrm{Oz}^{1}$ \\ ${ }^{1}$ Department of Pathology, Cerrahpasa Faculty of Medicine, Istanbul University, 34000 Istanbul, Turkey \\ ${ }^{2}$ Department of Radiology, Faculty of Medicine, Yeditepe University, Istanbul, Turkey \\ Correspondence should be addressed to Nil Comunoglu; nilustundag@yahoo.com
}

Received 16 October 2014; Accepted 14 November 2014; Published 10 December 2014

Academic Editor: Cheryl Ann Palmer

Copyright (c) 2014 Nil Comunoglu et al. This is an open access article distributed under the Creative Commons Attribution License, which permits unrestricted use, distribution, and reproduction in any medium, provided the original work is properly cited.

\begin{abstract}
Glioneuronal neoplasms are rare tumors. Recently, an unusual glioneuronal tumor histologically showing neuropil-like islands has been described. Here, we present such a tumor originating from spinal cord of a 14 -year-old girl, who has scoliosis and urinary incontinence. Microscopically, the glial component was chiefly fibrillary astrocytic, punctuated by neuropil-like islands. Immunohistochemically, glial tissue was GFAP positive, and neuropil-like areas and big neurons were synaptophysin reactive. For astrocytic component $\mathrm{Ki}-67$ proliferation index was $1 \%$ and p53 was immunonegative. This case is unique in that in the literature it is the second reported case in pediatric age group that is located at spinal cord.
\end{abstract}

\section{Introduction}

Three new entities have been recently added to the group of glioneuronal tumors by the recently updated World Health Organization (WHO) Classification of Tumors of Central Nervous System [1]. Evolution of this classification has been achieved by recent positive results of immunohistochemical and molecular studies. The new entities are papillary glioneuronal tumor (PGT), rosetted glioneuronal tumor with neuropil-like islands, and rosette-forming glioneuronal tumor (RGNT) of fourth ventricle. These tumors, morphologically resembling glial neoplasms, have readily been identified by neuronal differentiation [1-3]. In particular, rosetted glioneuronal tumor with neuropil-like islands should be distinguished from RGNT and PGT. The lesion currently is considered as a variant of astrocytomas, WHO Grade II or Grade III [1]. In 1999, Teo et al. reported a series of 4 cases of a glioneuronal tumor of the adult cerebrum that were marked by neuropil-like or rosetted islands, otherwise resembling diffusely infiltrating astrocytomas [4]. Most cases in the literature have been located in the cerebrum [2-8]. Spinal cord localization has been reported very rarely [9-11]. The present case is the second rosetted glioneuronal tumor with neuropil-like islands occurring in childhood period localized at spinal cord.

\section{Case Report}

2.1. Clinical Summary. We present a 14-year-old female patient followed up because of her scoliosis and urinary incontinence. Cervical and thoracic magnetic resonance imaging (MRI), performed during follow-up of her scoliosis, revealed a cervical intrathoracic mass lesion. The patient was then referred to the neurosurgery department. MRI revealed that an intramedullary mass lesion containing solid and cystic components was detected located at $\mathrm{C} 5-\mathrm{T} 5$, resulting in cervical scoliosis with right concave side and an expansion in spinal canal. Solid component of the mass was extending through C7-T3 vertebrae and was measuring $51 \times 18 \times 22 \mathrm{~mm}$. This solid component of the tumor was heterogeneously hypointense on $\mathrm{T} 1$ image and heterogeneously hyperintense on T2 image (Figures 1 and 2). Surgically, C5, 6, 7 and T1 hemilaminotomy and intradural intramedullary tumor excision were performed.

2.2. Pathological Findings. To the pathology department, yellowish 11 irregular tissue fragments, with the greatest measuring $6 \times 4 \times 2 \mathrm{~mm}$, were submitted. Microscopic examination showed a mixed glioneuronal tumor composed of oligodendrocyte-like cells constituting neuropil-like islands, 


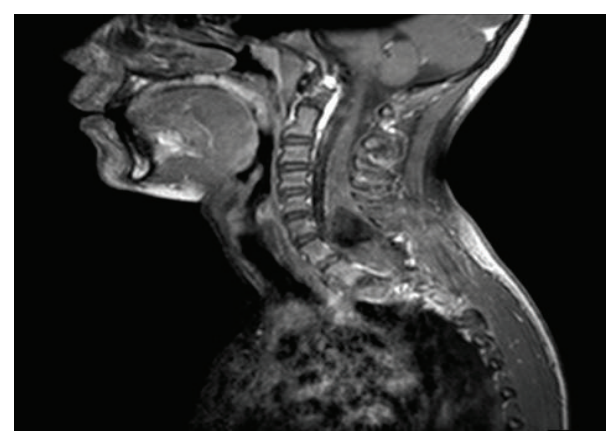

FIGURE 1: Sagittal fat saturated postcontrast spin-echo (SE) T1weighted MRI of the cervical spine demonstrates a large expansile mixed solid and cystic intra-axial mass of the spinal cord extending from C5 to T1 level. Both the upper cystic and the lower solid components do not enhance.

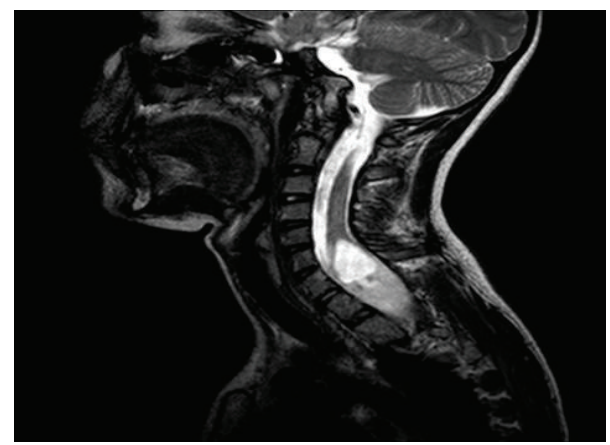

FIgURE 2: Sagittal turbo spin-echo (TSE) T2-weighted MRI of cervical spine demonstrates a large expansile intra-axial mass of the spinal cord. The upper cystic part and the lower solid parts of the mass are hyperintense.

astrocytic cells, and neurons scattered in glial tissue (Figures 3 and 4). Astrocytic component consisted of microcystic fibrillary regions which does not contain Rosenthal fibers. Astrocytic component of the tumor did not contain mitosis, vascular proliferation, or necrosis. The tumor, graded according to the astrocytic component, was considered as WHO Grade 2. Immunohistochemically, glial tissue was GFAP positive (clone 6F2, DAKO), and neuropil-like areas and big neurons were synaptophysin positive (clone SY38, DAKO) (Figure 5). In astrocytic component Ki-67 (clone MIB-1, DAKO) proliferation index was 1\%. p53 (clone 3186-11, DAKO) was immunonegative. Consequently, the final diagnosis was spinal cord glioneuronal tumor with rosetted neuropil-like islands-WHO Grade II.

\section{Discussion}

Our case is a childhood glioneuronal tumor with rosetted neuropil-like islands located at the cervicothoracic region of the spinal cord. This group of tumors was firstly described by Teo et al. [4]. These tumors usually present with supratentorial, hemispheric location. Reported patients are between the ages of 23 and 44 [2-8]. Harris and Horoupian, Rickert et al.,

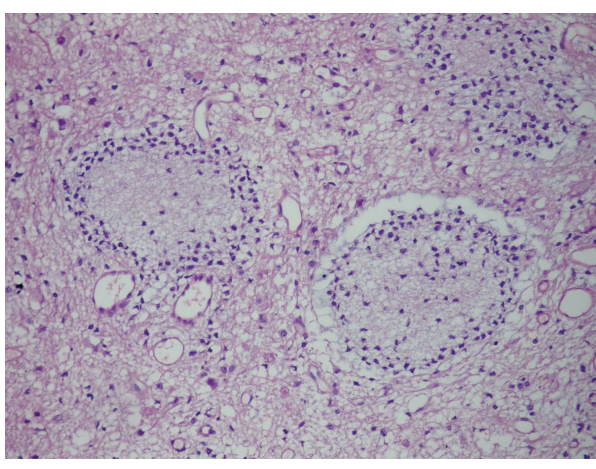

Figure 3: Oligodendrocyte-like cells and neuropil-like islands $(\mathrm{H} \& \mathrm{E} ; \times 200)$.

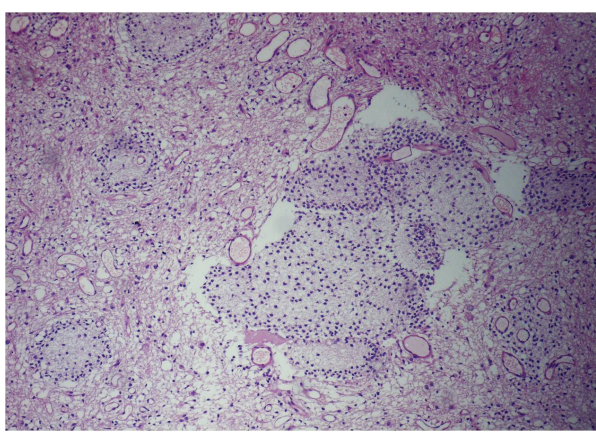

FIGURE 4: Neuropil-like island $(\mathrm{H} \& \mathrm{E} ; \times 100)$.

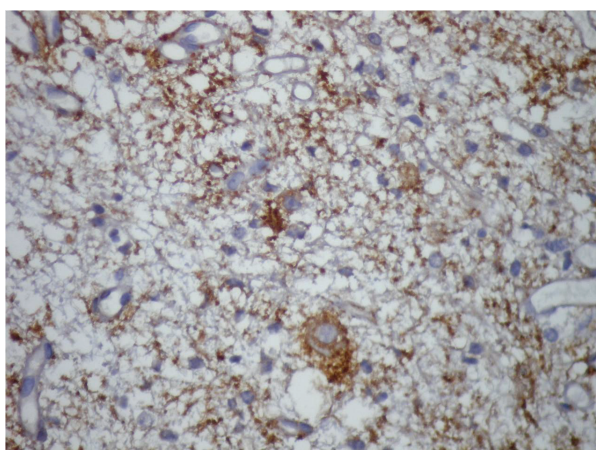

FIGURE 5: Synaptophysin positivity in large neurons (synaptophysin; $\times 400)$.

and Poliani et al. have reported cases of spinal cord location [9-11]. To the best of our knowledge, our case is the second youngest case having this tumor at the spinal cord.

Radiologically, intramedullary tumors are usually described as nodular, moderately contrast enhancing lesions. Cerebral cases are hypointense in $\mathrm{T} 1$ and hyperintense and focally contrast enhancing in T2. Hemispheric white matter, corpus callosum, and basal ganglia locations have been reported $[2-5,9]$.

These tumors are morphologically biphasic tumors composed of astrocytic and neuronal components. Distinct from diffuse astrocytomas, neuropil-like islands disperse in neuroparenchyma in permeative fashion. In some cases, 
astrocytic component may be dominant. Microcystic and myxoid changes can be detected within these regions. Cellular component of neuropil-like islands are oligodendrocytelike cells displaying perinuclear clearing. Big neurons are scattered in glial tissue. They do not show pleomorphism or binucleation [2-5].

Immunohistochemically, glial tissue is positive for GFAP; neuropil-like islands and big neurons are positive for neuronal markers such as synaptophysin, $\mathrm{Neu}-\mathrm{N}$, and $\mathrm{Hu}$ [1]. Keyvani et al. reported that, within these tumors, Ki-67 proliferation index was 3.9\% (0.6-9.3) in neuronal component and $1.5 \%(0.6-2.6)$ in astrocytic component [3]. In our case, Ki-67 proliferation index was $1 \%$ in astrocytic component and it was negative in neuronal component. Positivity for p53 has been reported in some cases [5]. p53 was negative in our case.

The glioneuronal tumors can be mistakenly diagnosed as oligodendrogliomas, astrocytomas, or ependymomas. Oligodendrogliomas are distinct with their molecular genetic features. Rarely, ependymomas containing neuropil-like islands have been reported [1]. Ependymomas can be distinguished morphologically by displaying formation of rosettes and pseudorosettes and immunohistochemically by EMA positivity. Perry et al. claimed that glioneuronal tumors were oligodendroglial tumors showing neurocytic differentiation [12]. Oligodendrocyte-like cells having perinuclear halo and $1 p$ 19q deletion in a small number of cases are histologic and molecular genetic findings, respectively, supporting Perry and his colleagues' claim [12,13]. However, it has been noted that in some cases $1 \mathrm{p} \mathrm{19q}$ deletion could not be detected [8]. On the contrary, Keyvani et al. found increase in $7 \mathrm{q}$ and loss in $9 p$ in molecular genetic studies of a small number of cases [3]. Loss of $4 q, 5 q$, and $11 p$ and gain of $6 p, 7 q, 8 q, 11 q, 12 p$, and $15 q$ were found in another cytogenetic study of Min et al. [14].

Actually these tumors can be diagnosed morphologically straightforwardly. They are classified within the group of astrocytomas; however, their category can be changed in the future classifications. Their rarity results in classification difficulties. It would be beneficial to collect cases in a center in order to conduct molecular studies and report the findings.

\section{Conflict of Interests}

The authors declare that there is no conflict of interests regarding the publication of this paper.

\section{References}

[1] D. Louis, H. Ohgaki, O. Wiestler, and W. Cavenee, WHO Classification of Tumours of the Central Nervous System, IARC, Lyon, France, 2007.

[2] V. Barbashina, P. Salazar, M. Ladanyi, M. K. Rosenblum, and M. A. Edgar, "Glioneuronal tumor with neuropil-like islands (GTNI): a report of 8 cases with chromosome 1p/19q deletion analysis," American Journal of Surgical Pathology, vol. 31, no. 8, pp. 1196-1202, 2007.

[3] K. Keyvani, C. H. Rickert, K. von Wild, and W. Paulus, "Rosetted glioneuronal tumor: a case with proliferating neuronal nodules," Acta Neuropathologica, vol. 101, no. 5, pp. 525-528, 2001.
[4] J. G. C. Teo, S. H. Gultekin, M. Bilsky, P. Gutin, and M. K. Rosenblum, "A distinctive glioneuronal tumor of the adult cerebrum with neuropil- like (including "rosetted") islands: report of 4 cases," The American Journal of Surgical Pathology, vol. 23, no. 5, pp. 502-510, 1999.

[5] R. A. Prayson and C. M. Abramovich, "Glioneuronal tumor with neuropil-like islands," Human Pathology, vol. 31, no. 11, pp. 1435-1438, 2000.

[6] E. F. Bisson, W. W. Pendlebury, and M. A. Horgan, "Glioneuronal tumor with unique imaging and histologic features," Journal of Neuro-Oncology, vol. 72, no. 1, pp. 89-90, 2005.

[7] I. Vajtai and M. M. Reinert, "Malignant glioneuronal tumor of the adult cerebrum with neuropil-like islands involving proliferating nodules: confirmatory report of an unusual variant," Acta Neuropathologica, vol. 113, no. 6, pp. 711-713, 2007.

[8] S. Agarwal, V. Suri, A. Rishi et al., "Glioneuronal tumor with neuropil-like islands: a new entity," Neuropathology, vol. 29, no. 1, pp. 96-100, 2009.

[9] B. T. Harris and D. S. Horoupian, "Spinal cord glioneuronal tumor with "rosetted" neuropil islands and meningeal dissemination: a case report," Acta Neuropathologica, vol. 100, no. 5, pp. 575-579, 2000.

[10] C. H. Rickert, M. Jasper, A. Sepehrnia, and A. Jeibmann, "Rosetted glioneuronal tumour of the spine: clinical, histological and cytogenetic data," Acta Neuropathologica, vol. 112, no. 2, pp. 231233, 2006.

[11] P. L. Poliani, D. Sperli, S. Valentini et al., "Spinal glioneuronal tumor with neuropil-like islands and meningeal dissemination: histopathological and radiological study of a pediatric case," Neuropathology, vol. 29, no. 5, pp. 574-578, 2009.

[12] A. Perry, B. W. Scheithauer, R. J. B. Macaulay, C. Raffel, K. A. Roth, and J. M. Kros, "Oligodendrogliomas with neurocytic differentiation. a report of 4 cases with diagnostic and histogenetic implications," Journal of Neuropathology \& Experimental Neurology, vol. 61, no. 11, pp. 947-955, 2002.

[13] T. J. Fraum, S. Barak, S. Pack et al., "Spinal cord glioneuronal tumor with neuropil-like islands with $1 \mathrm{p} / 19 \mathrm{q}$ deletion in an adult with low-grade cerebral oligodendroglioma," Journal of NeuroOncology, vol. 107, no. 2, pp. 421-426, 2012.

[14] H. S. Min, S. H. Lee, H. Yoo, J. Myung, E. K. Hong, and S.-H. Park, "Cytogenetic study of glioneuronal tumor with neuropillike islands: a case report," Neuropathology, vol. 30, no. 4, pp. 420-426, 2010. 


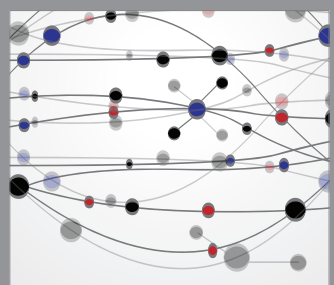

The Scientific World Journal
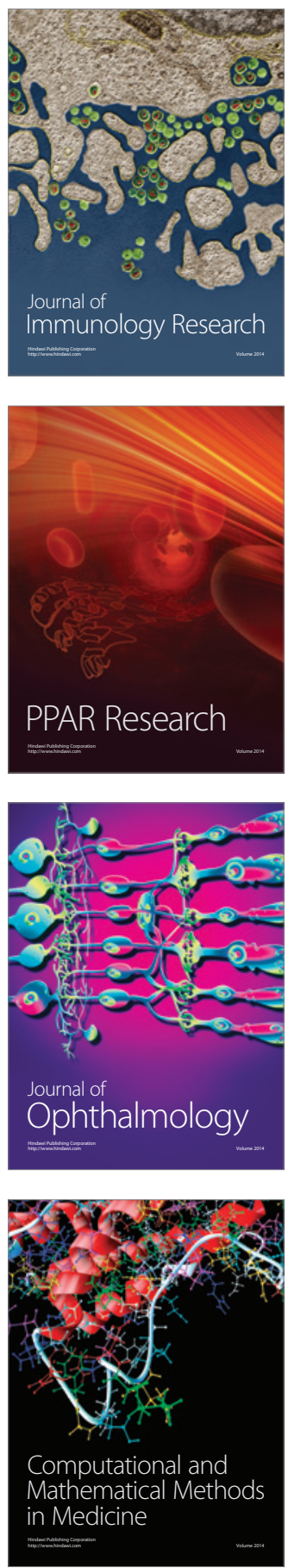

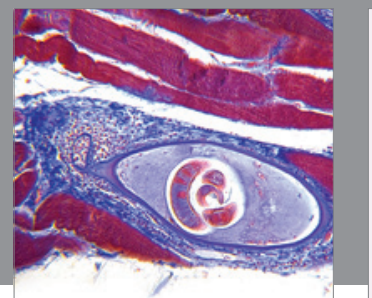

Gastroenterology

Research and Practice
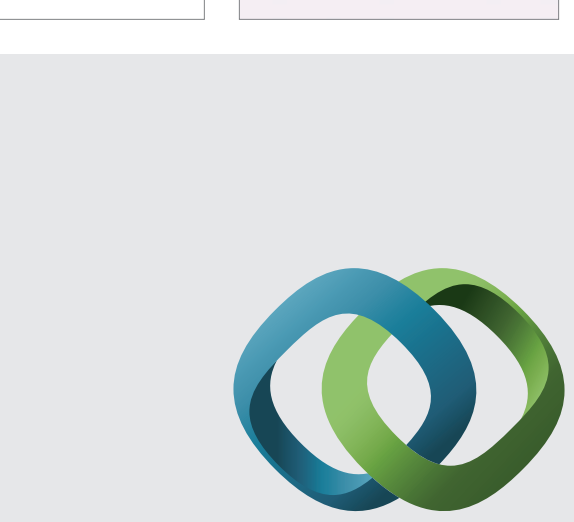

\section{Hindawi}

Submit your manuscripts at

http://www.hindawi.com
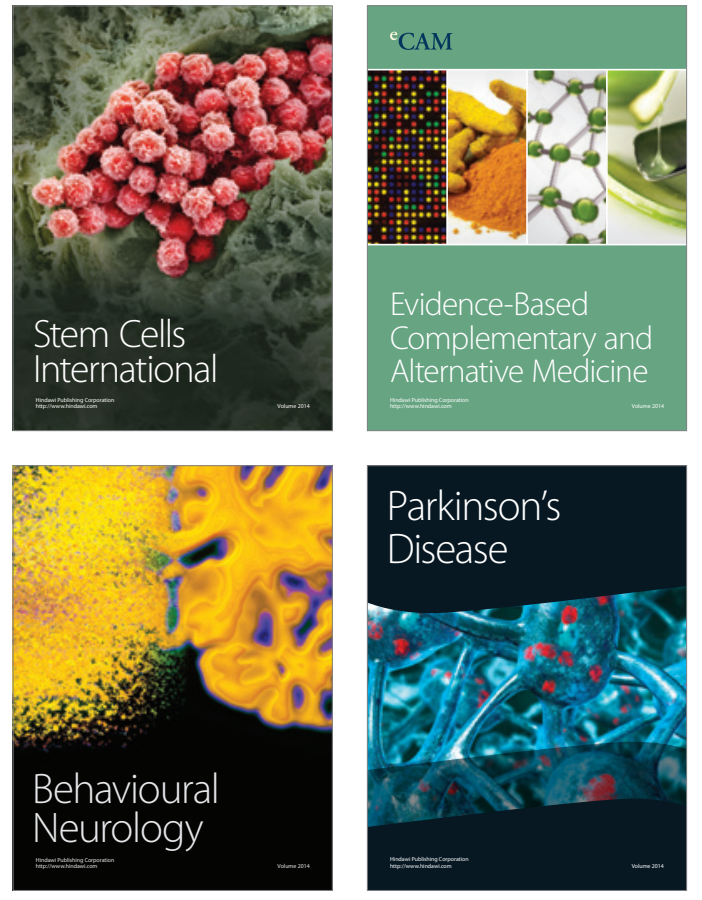
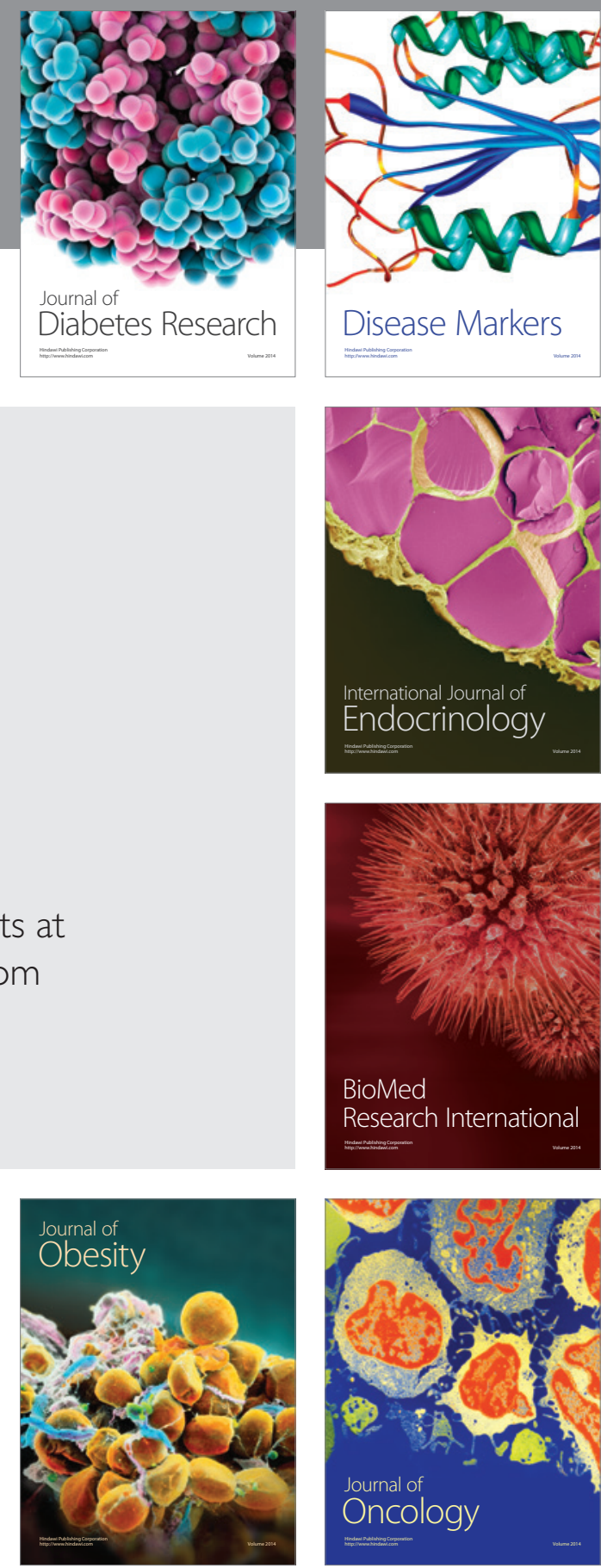

Disease Markers
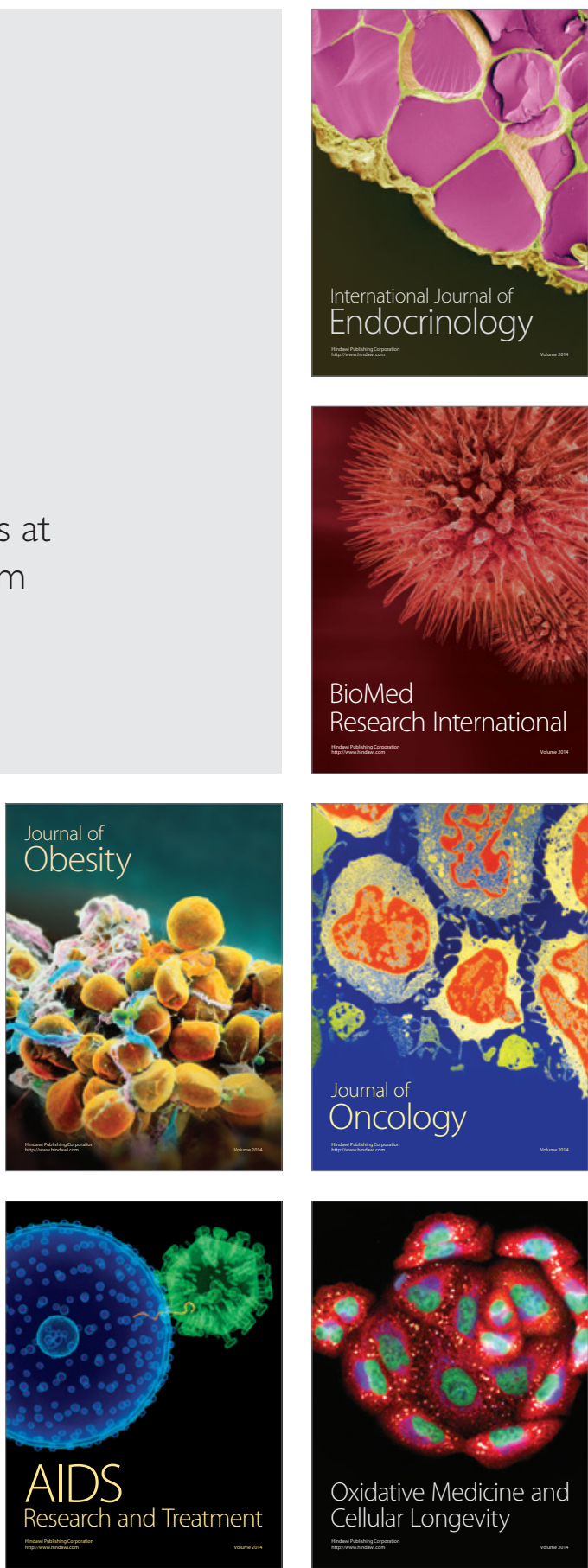